\title{
SHORT COMMUNICATION \\ ENVIRONMENTAL TOBACCO SMOKE: IS IT A RISK FACTOR FOR DIARRHEA IN 6-18 MONTHS OLD INFANTS?
}

\author{
Elif N. Özmert, Mustafa Kiliç, Kadriye Yurdakök \\ Hacettepe University Faculty of Medicine, Department of Pediatrics, Social Pediatrics Unit, Ankara, Turkey
}

\begin{abstract}
SUMMARY
Environmental tobacco smoke (ETS) has been shown to have many untoward healh effects, however no study directly evaluated the effect of ETS on diarrheal diseases. In this case control study we aimed to define the risk factors including ETS for diarrheal diseases in 6-18 months old infants who live in the capital of Turkey. All of them have access to pipe water and toilets. Infants who were admitted to Hacettepe University Insan Dogramaci Children's Hospital Diarrheal Diseases Training and Treatment Unit with the complaint of acute diarrhea (less than 14 days) between April-May 2005 constituted the cases. Controls were age matched infants who were admitted to Well Baby Unit during the same period and never had a diarrheal episode. A total of 206 children were included in the study (104 were diarrheal cases and 102 control cases). The only statistically significant parameters were found to be maternal education (OR:2.7 95\%Cl 1.2-5.9, $p=0.014)$, maternal cigarette smoking (OR:2.9, $95 \% \mathrm{Cl}: 1.2-6.9, p=0.015)$ and infant's weight for height being below 10 percentile (OR:2.2, 95\%Cl:1.1-4.5, $p=0.045)$. ETS should be evaluated as a risk factor for diarrhea in further studies.
\end{abstract}

Key words: diarrhea, infant, environmental tobacco smoke

Address for correspondence: E. N. Özmert, Hacettepe University Faculty of Medicine, Department of Pediatrics, Social Pediatrics Unit, 06100 Ankara, Turkey. E-mail: nozmert@hacettepe.edu.tr

Diarrheal diseases fall among the important causes of childhood morbidity and mortality. Hygiene and sanitary measures (toilets, water) are important factors contributing to diarrheal diseases (1). However, diarrhea still affects children in societies with good sanitary conditions. Although environmental tobacco smoke (ETS) has been shown to have many untoward healh effects no study directly evaluated the effect of ETS on diarrheal diseases. Only two studies could be encountered relating indoor nitrogen dioxide levels with diarrheal diseases and in turn relating indoor nitrogen dioxide levels to cigarette smoking (2).

In this case control study we aimed to define the risk factors including ETS for diarrheal diseases in 6-18 months old infants who are living in the capital of Turkey and all have access to pipe water and toilets.

Infants who were admitted to Hacettepe University Ihsan Dogramaci Children's Hospital Diarrheal Diseases Training and Treatment Unit with the complaint of acute diarrhea (less than 14 days) between April-May 2005 constituted the cases. Controls were age matched infants admitted to Well Baby Unit during the same period who never experienced a diarrheal episode. Oral informed consent was taken from all families and a questionaire was distributed. Routine stool culture for only Salmonella and
Shigella are made in our Center. None of the cases in the study group revealed culture positivity for these microorganisms.

Statistical analysis was performed by SPSS 10.0. Univariate analysis was performed by Student's t-test and $\chi^{2}$ tests when appropriate. Variables found statistically significant in univariate analysis were included into the multivariate logistic regression model.

A total of 206 children were included in the study. 104 were diarrheal cases and 102 control cases. The characteristics of cases are displayed in Table 1 . The mean age $(11.9 \pm 3.82$ vs $11.2 \pm 3.80$, $\mathrm{p}>0.05$, values are "mean \pm SD") was similar in both groups. The paramaters that were statistically significantly different between the groups were included into the multivariate logistic model. The results are displayed in Table 2 . The only statistically significant parameters were found to be maternal education (OR:2.7 95\%CI 1.2-5.9, $\mathrm{p}=0.014$ ), maternal cigarette smoking (OR:2.9, 95\%CI:1.2-6.9, $\mathrm{p}=0.015)$ and infant's weight for height being below 10 percentile (OR:2.2, 95\%CI:1.1-4.5, $\mathrm{p}=0.045$ ).

In this study a case control method was used to determine risk factors for diarrhea. As the study population was 6-18 months old it is believed that memory bias would be minimal. Maternal education and low weight for height percentile were found to be 
Table 1. Comparison of risk factors for diarrhea among 6-18 months old infants

\begin{tabular}{|l|c|c|c|c|}
\hline Characteristic & $\begin{array}{c}\text { Diarrhea Cases } \\
(\mathrm{N}=104)\end{array}$ & $\begin{array}{c}\text { Control Cases } \\
(\mathrm{N}=102)\end{array}$ & OR (95 \% Cl) & $\mathbf{p}$ \\
\hline Sex (F/M) & $41 / 63$ & $51 / 51$ & $0.6(0.4-1.1)$ & 0.06 \\
\hline $\begin{array}{l}\text { Maternal education } \\
<8 \text { years }\end{array}$ & $29(27.8)$ & $14(13.7)$ & $2.3(1.1-4.6)$ & 0.015 \\
\hline $\begin{array}{l}\text { Paternal education } \\
<8 \text { years }\end{array}$ & $8(7.7)$ & $3(2.9)$ & $2.6(0.6-9.9)$ & 0.137 \\
\hline Current breast-feeding & $54(51.9)$ & $73(71.6)$ & $0.4(0.2-0.7)$ & 0.002 \\
\hline Bottle feeding & $70(67.3)$ & $56(54.9)$ & $1.8(1.01-3.2)$ & 0.025 \\
\hline Pacifier use & $37(35.6)$ & $39(38.2)$ & $0.9(0.55-1.7)$ & 0.521 \\
\hline Regular yogurt consumption & $80(76.9)$ & $79(77.4)$ & $0.6(0.3-1.3)$ & 0.156 \\
\hline Weight for height <10 p & $30(28.8)$ & $17(16.7)$ & $2.2(1.1-4.3)$ & 0.015 \\
\hline$<4$ months complementary feeding & $41(39.4)$ & $27(26.5)$ & $1.8(1.02-3.3)$ & 0.030 \\
\hline Maternal smoking & $28(26.9)$ & $13(12.7)$ & $2.4(1.1-4.9)$ & 0.013 \\
\hline Smoking during pregnancy & $13(12.5)$ & $6(5.9)$ & $2(0.8-6.1)$ & $1.2(0.7-2.1)$ \\
\hline Paternal smoking & $48(46.1)$ & $42(41.2)$ & $0.9(0.6-1.6)$ & 0.087 \\
\hline Having at least one sibling & $59(56.7)$ & $55(53.9)$ & 0.323 \\
\hline
\end{tabular}

Table 2. Multivariate logistic regression of the risk factors for diarrhea among 6-18 months old infants*

\begin{tabular}{|l|c|c|c|}
\hline Characteristic & Odds Ratio & $95 \% \mathrm{Cl}$ & $\mathrm{p}$ \\
\hline Maternal education < 8 years & 2.7 & $1.2-5.9$ & 0.014 \\
\hline Current breast feeding & 0.5 & $0.3-1.1$ & 0.079 \\
\hline Bottle feeding & 1.4 & $0.7-2.8$ & 0.303 \\
\hline Weight for height <10 $\mathrm{p}$ & 2.2 & $1.1-4.5$ & 0.045 \\
\hline Maternal smoking & 2.9 & $1.2-6.9$ & 0.015 \\
\hline$<4$ months complementary feeding & 1.6 & $0.8-3.1$ & 0.181 \\
\hline
\end{tabular}

*: all variables are categorical, interactions are not involved

risk factors as in many other studies $(1,3,4)$. A new risk factor found to be associated with diarrhea in this study was maternal cigarette smoking.

The effect of ETS has not been studied much as a risk factor for diarrheal diseases in earlier reports. We could find only two related reports from UK investigating the correlation between indoor nitrogen oxide (NO) compounds and child health. They have reported that indoor levels of NO compounds correlated significantly with diarrhea in both term and preterm born infants. ETS was found to be an important contributor to indoor levels of NO compounds (2). The pathophysiology was speculated to be related either to the disruption of the mucosal barrier function or diarrheogenic effect of NO compounds $(2,5)$. Of course the relation of ETS and diarrhea may be related to other factors (decreased antioxidants, etc.) also. It is suggested that the effect of ETS as a risk factor for diarrhea deserves further studies.

\section{REFERENCES}

1. Mølbak K, Jensen H, Ingholt L, Aaby P. Risk factors for diarrheal disease incidence in early childhood: a community cohort study from GuineaBissau. Am J Epidemiol. 1997 Aug 1;146(3):273-82.

2. Farrow SC, Farrow A. Diarrhoea and nitrogen oxides. Med Hypotheses. 1999 Sep;53(3):224-31.

3. Basu AM, Stephenson R. Low levels of maternal education and the proximate determinants of childhood mortality: a little learning is not a dangerous thing. Soc Sci Med. 2005 May;60(9):2011-23.

4. Victora CG, Barros FC, Kirkwood BR, Vaughan JP. Pneumonia , diarrhea and growth in the first 4 y of life: a longitudinal study of 5914 urban Brazilian children. Am J Clin Nutr. 1990 Aug;52(2):391-6.

5. Fasano A. Toxins and the gut: role in human disease. Gut. 2002 May;50 Suppl 3:III9-14. 